\title{
ON HILBERT SPACES WITH UNITAL MULTIPLICATION
}

\author{
BORUT ZALAR \\ (Communicated by Palle E. T. Jorgensen)
}

\begin{abstract}
We give a new simplified proof of two theorems of Froelich, Ingelstam, and Smiley. Our approach enables us also to generalize both of them. In the second section we prove a related theorem which requires different methods for its proof.
\end{abstract}

\section{INTRODUCTION}

The study of strictly cyclic operator algebras due to John Froelich pointed out associative Hilbert algebras with identity 1 satisfying $|x y| \leq|x||y|$ and $|1|=1$ where $|x|=\sqrt{\langle x, x\rangle}$ is a norm derived from the inner product. These algebras were already studied by Ingelstam in [2] who used the analysis of the so called vertex property for Banach algebras. He proved that such algebras are necessarily division algebras.

A simpler proof was given by Smiley in [3] and his proof was in turn greatly simplified by Froelich in his recent paper [1] which is a base point for our investigation. Our paper has three goals:

(i) Froelich used in his proof Gelfand theory and the Riesz representation theorem. As we show even those can be avoided in order to obtain probably the simplest possible proof. $|x|^{2}$.

(ii) We shall replace original assumption $|x y| \leq|x||y|$ by a weaker one $\left|x^{2}\right| \leq$

(iii) In some of our results we can avoid the assumption of associativity.

Let $\mathbb{R}, \mathbb{C}, \mathbb{H}$, and $\mathbb{D}$ denote real numbers, complex numbers, quaternions, and octonions, respectively.

\section{Generalizations of Froelich-Ingelstam-Smiley theorems}

Proposition 1. Let $\mathscr{A}$ be a real nonassociative pre-Hilbert algebra with identity 1 , and suppose that $\left|a^{2}\right| \leq|a|^{2}$ holds for all $a \in \mathscr{A}$ and $|1|=1$. Then for every nonzero $a \in \mathscr{A}$ there exists $a^{*} \in \mathscr{A}$ such that $a a^{*}=a^{*} a=1$.

Received by the editors June 9, 1993 and, in revised form, August 24, 1993.

1991 Mathematics Subject Classification. Primary 46K15; Secondary 46H70.

Key words and phrases. Hilbert space, Hilbert algebra, absolute valued algebra, division algebra, topological zero divisor. 
Proof. Suppose that we have $x \in\{1\}^{\perp}$ with $|x|=1$. For each $\lambda \in \mathbb{R}$ we have

$$
\left|(\lambda+x)^{2}\right|^{2}=\left|\lambda^{2}+2 \lambda x+x^{2}\right|^{2} \leq|\lambda+x|^{4}
$$

and so

$$
2 \lambda^{2}\left(1+\left\langle 1, x^{2}\right\rangle\right)+4 \lambda\left\langle x, x^{2}\right\rangle+\left|x^{2}\right|^{2}-1 \leq 0 .
$$

This is possible for all real $\lambda$ only if $1+\left\langle 1, x^{2}\right\rangle \leq 0$. On the other hand

$$
\left|\left\langle 1, x^{2}\right\rangle\right| \leq|1|\left|x^{2}\right| \leq|x|^{2}=1
$$

and so $x^{2}=-1$ follows. If $x \in\{1\}^{\perp}$ is arbitrary, then $x^{2}=-|x|^{2}$ follows. Note that this trivially holds for $x=0$ as well.

Given a nonzero $a \in \mathscr{A}$ we may decompose $a=\lambda+x$ where $\lambda \in \mathbb{R}$ and $x \in\{1\}^{\perp}$. Since $a \neq 0$, we have $\lambda^{2}+|x|^{2}=|a|^{2} \neq 0$ and so we may define $a^{*}=\frac{1}{\lambda^{2}+|x|^{2}}(\lambda-x)$. Using the above paragraph, we can easily compute $a a^{*}=a^{*} a=1$.

If we use Proposition 1 and the well-known fact that every associative division normed algebra is isomorphic to $\mathbb{R}, \mathbb{C}$, or $\mathbb{H}$, we obtain

Corollary 1 (the first Froelich-Ingelstam-Smiley theorem). Let $\mathscr{A}$ be a real associative pre-Hilbert algebra with identity 1 , and suppose that $|a b| \leq|a||b|$ holds for all $a, b \in \mathscr{A}$ and $|1|=1$. Then $\mathscr{A}$ is isomorphic to $\mathbb{R}, \mathbb{C}$, or $\mathbb{H}$.

However if we base our proof on the concept of the absolute valued algebra rather than on division normed algebras, then the closer inspection of the proof of Proposition 1 gives us the following generalization of Corollary 1:

Theorem 1. Let $\mathscr{A}$ be alternative real pre-Hilbert algebra with identity 1. Suppose that $\left|a^{2}\right| \leq|a|^{2}$ holds for all $a \in \mathscr{A}$ and $|1|=1$. Then $\mathscr{A}$ is isomorphic to $\mathbb{R}, \mathbb{C}, \mathbb{H}$, or $\mathbb{D}$.

Proof. Let us recall first that algebra is called alternative if $a^{2} b=a(a b)$ and $b a^{2}=(b a) a$ for all $a, b \in \mathscr{A}$. Every associative algebra is obviously alternative while $\mathbb{D}$ is alternative but not associative.

Next we recall from the proof of Proposition 1 that for each $x \in\{1\}^{\perp}$ the equality $x^{2}=-|x|^{2}$ holds. This implies that $\left|a^{2}\right|=|a|^{2}$ in fact holds for all $a \in \mathscr{A}$ since, if we decompose $a=\lambda+x$,

$$
\left|a^{2}\right|=\left.\left.\left|\lambda^{2}+2 \lambda x-\right| x\right|^{2}\left|=\sqrt{\left(\lambda^{2}-|x|^{2}\right)^{2}+4 \lambda^{2}|x|^{2}}=\lambda^{2}+\right| x\right|^{2}=|a|^{2} .
$$

In our first step we shall assume that $1, x, y$ are pairwise orthogonal. Then

$$
(x+y)^{2}=-|x+y|^{2}=-|x|^{2}-|y|^{2}=x^{2}+y^{2}
$$

implies $x y=-y x$. This further implies, together with the Moufang identity $x y \cdot y x=x \cdot y^{2} \cdot x$ which is valid in every alternative algebra,

$$
|x y|^{2}=\left|(x y)^{2}\right|=|x y \cdot x y|=|x y \cdot y x|=\left|x y^{2} x\right|=|x|^{2}|y|^{2} .
$$

In our second step we shall take $x, y$ both orthogonal to 1 . In the same way as in the above paragraph we can verify $x y+y x=-2\langle x, y\rangle$. Decompose $x y=$ $\langle 1, x y\rangle+z$ and $y x=\langle 1, y x\rangle+z_{1}$. Since $x y+y x \in \mathbb{R} 1$ and $z+z_{1} \in\{1\}^{\perp}$, we have $z_{1}=-z$. From $x(x y)=x^{2} y=-|x|^{2} y$ we obtain $\langle 1, x y\rangle x+x z=$ $-|x|^{2} y$. From $(y x) x=y x^{2}=-|x|^{2} y$ we obtain

$$
\langle 1, y x\rangle x-z x=-|x|^{2} y=\langle 1, x y\rangle x+x z .
$$


But $x z+z x=-2\langle x, z\rangle \in \mathbb{R} 1$ while $x \in\{1\}^{\perp}$, so we have $\langle 1, x y\rangle=\langle 1, y x\rangle$ and $\langle z, x\rangle=0$. Therefore

$$
\begin{gathered}
\langle 1, x y\rangle=\langle 1, y x\rangle=-\langle x, y\rangle, \\
\langle x y, x\rangle=\langle y x, x\rangle=0
\end{gathered}
$$

if $x, y \in\{1\}^{\perp}$. Now we shall prove that $|x y|=|x||y|$. If $x=0$, then the result is trivial. Otherwise define

$$
y_{1}=\frac{-\langle x, y\rangle}{|x|^{2}} x+y
$$

so that $x$ is orthogonal to $y_{1}$. According to the above paragraph, we have $\left|x y_{1}\right|=|x|\left|y_{1}\right|$. Thus

$$
|\langle x, y\rangle+x y|^{2}=|x|^{2}\left(|y|^{2}-\frac{\langle x, y\rangle^{2}}{|x|^{2}}\right)=|x|^{2}|y|^{2}-\langle x, y\rangle^{2} .
$$

According to (1), we have

$$
|\langle x, y\rangle+x y|^{2}=\langle x, y\rangle^{2}+2\langle x, y\rangle\langle 1, x y\rangle+|x y|^{2}=|x y|^{2}-\langle x, y\rangle^{2}
$$

and finally $|x y|=|x||y|$.

In our last step we take any $a, b \in \mathscr{A}$ and decompose $a=\lambda+x, b=\mu+y$. Then

$$
\begin{aligned}
|a|^{2}|b|^{2}-|a b|^{2}= & \lambda^{2} \mu^{2}+\lambda^{2}|y|^{2}+\mu^{2}|x|^{2} \\
& +|x|^{2}|y|^{2}-\lambda^{2} \mu^{2}-\lambda^{2}|y|^{2}-\mu^{2}|x|^{2}-|x y|^{2} \\
& -2 \lambda \mu(\langle 1, x y\rangle+\langle x, y\rangle)-2 \lambda\langle y, x y\rangle-2 \mu\langle x, x y\rangle,
\end{aligned}
$$

so, by (1) and (2), it follows that $|a b|=|a||b|$. Thus $\mathscr{A}$ is an absolute valued algebra with identity and consequently isomorphic to $\mathbb{R}, \mathbb{C}, \mathbb{H}$, or $\mathbb{D}$.

Theorem 2 (generalization of the second Froelich-Ingelstam-Smiley theorem). Let $\mathscr{A}$ be a nonassociative complex pre-Hilbert algebra with identity 1 , and suppose that $\left|a^{2}\right| \leq|a|^{2}$ holds for all $a \in \mathscr{A}$ and $|1|=1$. Then $\mathscr{A}$ is isomorphic to $\mathbb{C}$ and is consequently automatically associative.

Proof. If $\mathscr{A}$ were not isomorphic to $\mathbb{C}$, then it would be at least two-dimensional over $\mathbb{C}$ and so there would exist some $x \in\{1\}^{\perp}$ with $|x|=1$. If we define a real inner product on $\mathscr{A}$ by $\langle a, b\rangle_{1}=\operatorname{Re}\langle a, b\rangle$, then $\mathscr{A}$ with this new inner product satisfies the assumptions of Proposition 1. Moreover $x$ and $i x$ are both orthogonal to 1 and so $x^{2}=(i x)^{2}=-1$ should hold which is clearly impossible.

We shall finish this section with an example which throws some light on the nonassociative case.

Example 1. Let $\mathscr{H}$ be a Hilbert space with dimension greater than one, and define the multiplication in $\mathscr{A}=\mathbb{R} \oplus \mathscr{H}$ by

$$
(\alpha \oplus x)(\beta \oplus y)=(\alpha \beta-\langle x, y\rangle) \oplus(\alpha y+\beta x)
$$

and the inner product by

$$
\langle(\alpha \oplus x),(\beta \oplus y)\rangle=\alpha \beta+\langle x, y\rangle .
$$


Then $\mathscr{A}$ satisfies $|a b| \leq|a||b|$ and $|1|=1$. However $\mathscr{A}$ contains divisors of zero, and therefore the existence of $a^{*}$ which satisfies $a a^{*}=a^{*} a=1$ (see Proposition 1) is not a sufficiently restrictive condition in the general nonassociative case. We do not see an easy way to describe all nonassociative algebras satisfying the assumptions of Proposition 1. Note that Example 1 is well known in the theory of Jordan algebras.

\section{Algebras SATISFYing $\left|x^{2}\right|=|x|^{2}$}

It is obvious that we cannot drop the existence of an identity element in the Froelich-Ingelstam-Smiley theorems. We can in fact produce a very trivial example. If $\mathscr{A}$ is any pre-Hilbert space and we define $a b=0$ for all $a, b \in \mathscr{A}$, then $\mathscr{A}$ is associative, $|a b| \leq|a||b|$, but $\mathscr{A}$ is not isomorphic to one of the algebras from these theorems. It is the purpose of this section to prove that if we change the inequality $\left|x^{2}\right| \leq|x|^{2}$ to the strict equality, then the existence of identity can be dropped.

Theorem 3. Let $\mathscr{A}$ be a real associative pre-Hilbert algebra satisfying $\left|a^{2}\right|=|a|^{2}$ for all $a \in \mathscr{A}$. Then $\mathscr{A}$ is isomorphic to $\mathbb{R}, \mathbb{C}$, or $\mathbb{H}$.

Proof. First we shall assume that $\mathscr{A}$ is commutative. Then

$$
|\langle a, b\rangle| \leq|a b| \leq|a||b|
$$

holds for all $a, b \in \mathscr{A}$ and consequently $\mathscr{A}$ is a normed algebra.

In fact

$$
\begin{aligned}
|a+b|^{2} & =\left|(a+b)^{2}\right|=\left|a^{2}+b^{2}+2 a b\right| \\
& \leq\left|a^{2}\right|+\left|b^{2}\right|+|2 a b|=|a|^{2}+|b|^{2}+2|a b|
\end{aligned}
$$

implies $\langle a, b\rangle \leq|a b|$. If we replace $a$ by $-a$, we get $|\langle a, b\rangle| \leq|a b|$.

Now assume for a moment that $|a|=|b|=1$. Then

$$
\begin{aligned}
4|a b| & =\left|(a+b)^{2}-(a-b)^{2}\right| \\
& \leq\left|(a+b)^{2}\right|+\left|(a-b)^{2}\right|=|a+b|^{2}+|a-b|^{2}=4
\end{aligned}
$$

and so $|a b| \leq 1$. In the general case we can reason as follows:

If $a=0$ or $b=0$, then $|a b| \leq|a||b|$ is obvious. Otherwise $\left|\frac{a}{|a|} \cdot \frac{b}{|b|}\right| \leq 1$ and so (3) follows.

Now we shall use the well-known fact that a commutative associative real normed algebra without topological zero divisors is isomorphic to $\mathbb{R}$ or $\mathbb{C}$. Our next goal is therefore to prove that the algebra under consideration does not have any topological zero divisors.

Suppose that $|a|=1,\left|x_{n}\right|=1$, and $a x_{n} \rightarrow 0$. By (3) we have

$$
\left|\left\langle a, x_{n}\right\rangle\right| \leq\left|a x_{n}\right| \rightarrow 0 \text {. }
$$

Since $\mathscr{A}$ is associative,

$$
\left|\left\langle a^{2}, x_{n}^{2}\right\rangle\right| \leq\left|a^{2} x_{n}^{2}\right|=\left|\left(a x_{n}\right)^{2}\right|=\left|a x_{n}\right|^{2} \rightarrow 0 .
$$

If we compute $\left|a+x_{n}\right|$ in a direct way, we obtain

$$
\left|a+x_{n}\right|^{4}=\left(2+2\left\langle a, x_{n}\right\rangle\right)^{2} \rightarrow 4 \text {. }
$$


If we use the square multiplicativity of the norm, we obtain

$$
\begin{aligned}
\left|a+x_{n}\right|^{4} & =\left|\left(a+x_{n}\right)^{2}\right|^{2}=\left|a^{2}+2 a x_{n}+x_{n}^{2}\right|^{2} \\
& =\left|a^{2}\right|^{2}+4\left|a x_{n}\right|^{2}+\left|x_{n}^{2}\right|^{2}+4\left\langle a^{2}, a x_{n}\right\rangle+4\left\langle a x_{n}, x_{n}^{2}\right\rangle+2\left\langle a^{2}, x_{n}^{2}\right\rangle .
\end{aligned}
$$

Since

$$
\begin{gathered}
\left|a^{2}\right|^{2}=|a|^{4}=1, \quad\left|x_{n}^{2}\right|^{2}=1, \\
\left|\left\langle a^{2}, a x_{n}\right\rangle\right| \leq\left|a^{2}\right|\left|a x_{n}\right|=\left|a x_{n}\right| \rightarrow 0, \\
\left|\left\langle a x_{n}, x_{n}^{2}\right\rangle\right| \leq\left|a x_{n}\right|\left|x_{n}^{2}\right|=\left|a x_{n}\right| \rightarrow 0,
\end{gathered}
$$

we have (note that $\left\langle a^{2}, x_{n}^{2}\right\rangle \rightarrow 0$ was already established) that $\left|a+x_{n}\right|^{4} \rightarrow 2$ which contradicts the previously obtained fact.

Now that we proved the result for the commutative case, we can handle the noncommutative one by means of localization. Take some nonzero $b \in \mathscr{A}$. A subalgebra $\operatorname{Gen}(b)$, generated by $b$, is commutative and so it is isomorphic to $\mathbb{R}$ or $\mathbb{C}$. Note that it is trivial that this subalgebra also satisfies the assumptions of our theorem. In particular this subalgebra contains the identity element which we denote by $e$. Then $e$ is of course an idempotent of $\mathscr{A}$. According to Theorem 1 it remains to prove that $e$ is the identity of $\mathscr{A}$.

Given an arbitrary $a \in \mathscr{A}$ we have $e(a-e a)=0$ and $(a-a e) e=0$. Since $e \neq 0$, it remains to prove that $\mathscr{A}$ cannot contain any zero divisors. If $x y=0$ with $|x|=|y|=1$, then $|y x|^{2}=\left|(y x)^{2}\right|=|y x y x|=0$ and so $y x=0$. Thus

$$
|x+y|^{2}=\left|(x+y)^{2}\right|=\left|x^{2}+y^{2}\right|=|x-y|^{2}
$$

implies $|x+y|^{2}=2$. Next we have

$$
\begin{aligned}
4 & =|x+y|^{4}=\left|x^{2}+y^{2}\right|^{2}=\left|x^{2}\right|^{2}+\left|y^{2}\right|^{2}+2\left\langle x^{2}, y^{2}\right\rangle \\
& =|x|^{4}+|y|^{4}+2\left\langle x^{2}, y^{2}\right\rangle=2+2\left\langle x^{2}, y^{2}\right\rangle
\end{aligned}
$$

and so $\left\langle x^{2}, y^{2}\right\rangle=1$ implies $x^{2}=y^{2}$. But then

$$
1=|x|^{4}=\left|x^{2}\right|^{2}=\left|x^{4}\right|=\left|x^{2} y^{2}\right|=0
$$

is a contradiction.

\section{ACKNOWLEDGMENT}

The author wishes to express his gratitude to Professor Angel Rodriguez for his helpful comments.

\section{REFERENCES}

1. J. Froelich, Unital multiplications on a Hilbert space, Proc. Amer. Math. Soc. 117 (1993), 757-759.

2. L. Ingelstam, $A$ vertex property for algebras with identity, Math. Scand. 11 (1962), 22-32.

3. M. F. Smiley, Real Hilbert algebras with identity, Proc. Amer. Math. Soc. 16 (1965), 440-441.

University of LuUbljana, Institute of Mathematics, Jadranska 19, 61000 LuUbluana, SLOVENIJA

Current address: Faculty of Civil Engineering, Department of Basic Sciences, University of Maribor, Smetanova 17, 62000 Maribor, Slovenija

E-mail address: borut.zalar@uni-lj.si 